IN brief

\section{First mass spectrometry diagnostic approved in US}

The US Food and Drug Administration in August approved its first mass spectrometry (MS) diagnostic for clinical microbiology use. The VITEK MS, manufactured by the French multinational bioMérieux, based in Marcy-l'Etoile, uses matrix-assisted laser desorption/ionization time-of-flight MS to analyze the proteins of microorganisms and compare them to its vast database of signature profiles. In conventional microbial identification, microbes isolated from patients are subjected to more expensive and slower biochemical and molecular assays before clinicians can act on the information. Given a pure culture of a bacterium or fungus, the VITEK MS can pinpoint its identity at the species level in minutes, with the ability to perform nearly 200 tests at once. The platform is already approved for use in Europe and other countries, but was finally cleared in the US through the de novo pathway, which is used for low-risk devices of a new type that would not warrant premarket approval. A very similar rival platform, the Biotyper from Billerica, Massachusetts-based Bruker, is right behind bioMérieux, with a $510(\mathrm{k})$ application pending. According to Estée Torok of Addenbrooke's Hospital, Cambridge, UK, who uses the Bruker Biotyper, the technology has revolutionized microbe identification in the diagnostic laboratory. At a fraction of the price per identification of older methodology and producing comparable results, MSbased devices like VITEK and Biotyper, Torok predicts, may one day supplant the traditional identification workflow in most clinical settings.

\section{IN their words}

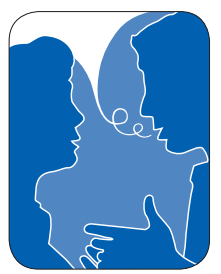

"In order to hit sales at rival companies and push the company's own products, bribes and special payments of all sorts were extremely common at the company. The level of the problem was just as bad as at GlaxoSmithKline." Wei Wang (pseudonym) former senior manager at Eli Lilly, which is alleged to have spent over $\$ 30$ million in bribes to Chinese physicians. (Reuters, 22 August 2013)

"It's that the provision of physician notification creates a psychological barrier to prescribing biosimilars." Tom Bone, legislative advocate for CVS Caremark pharmacies commenting on the possible impact of a biosimilar law under consideration by the California legislature. (California HealthLine, 22 August 2013)

\title{
Scripps boosts research coffers by selling reagents to Sigma-Aldrich
}

Six high-profile laboratories at The Scripps Research Institute have entered an alliance with Sigma-Aldrich that is the first of its kind for a chemical reagent supplier. Under the agreement announced in July, Sigma-Aldrich obtains an exclusive license to research reagents coming out of research laboratories led by Phil Baran, Jin-Quan Yu, Benjamin Cravatt, Carlos Barbas, Phillip Dawson and Barry Sharpless. The St. Louis-based SigmaAldrich will offer the Scripps researchers' cutting-edge drug discovery tools to its customers immediately after publication, in exchange for research support to Scripps. The transaction, which improves the coordination

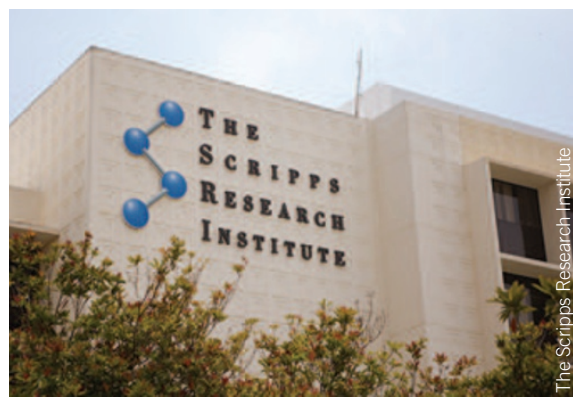

The Scripps Research Institute, home to Nobe prizewinners and pioneering scientists, is a nonprofit research institution whose philosophy is the pursuit of basic knowledge in life sciences for its application in medicine.

Sigma-Aldrich had previously established one-time relationships for specific reagents with the Sharpless laboratory (for reagents useful in performing 'click' chemistry, a platform that uses a set of reliable, selective reactions for the rapid synthesis of new compounds) and with Baran's group (to commercialize a toolkit of zinc salts including difluoromethanesulfinate, used to insert fluorine atoms directly into various organic substrates). But the multilaboratory scope of the new arrangement, which anticipates Sigma-Aldrich getting exclusive rights to classes of reagents not yet developed as well as those almost ready to roll out, is what makes this deal

between reagent discovery and commercialization, benefits the wider scientific community by enabling rapid access to the latest reagents. It also bolsters Scripps' research funding, an option that other academic institutions may find appealing in light of the new realities of research funding in the US.

Cutbacks in government grant support have made academic institutions like Scripps more flexible in considering commercial arrangements. The pharma-biotech industry has new balance-sheet concerns of its own and is turning to academia in new, more targeted ways as it seeks to externalize its R\&D. Thus, although La Jolla, California-based Scripps has a history of signing blanket 'first-rights' agreements with large drug companies, those firms are no longer offering such massive, open-ended research support.

Within this alliance, which has the structure and feel of an alliance between a pharmaceutical company and a not for profit, the Scripps teams will devise reagents and building blocks that improve the processes of medicinal chemistry and chemical biology, such as catalysts and probes and peptide-type chemistries. The Yu laboratory, for example, is focused on carbon-hydrogen bond-forming activation. Such bond creation is useful in improving catalytic reactions that parallel enzymatic transformations in terms of their reactivity and selectivity, and so is of interest to researchers building compound-screening libraries. the first agreement of this sort. "I don't think anybody has tapped a reagent company or allowed a reagent company to tap them in quite this way," says Scott Forrest, vice president of business development at Scripps. It's also the first time Sigma-Aldrich has made upfront investments for external reagent development.

Speeding time to market was a crucial consideration on both sides. There's usually a time delay between a publication showing the use of a reagent and its commercial availability of up to 12 months. But for the Scripps laboratories, products will be available at the time of publication.

"We believe that will help accelerate research into new areas of research," says Amanda Halford, vice president, academic research, at Sigma-Aldrich. The time of publication is when the most interest and awareness is generated. "It's the peak of demand for reagents," she says.

Having small quantities of reagents ready to go together with the publication references will also allow Sigma-Aldrich to get an early read on how commercially viable a reagent is and to jointly troubleshoot any issues of process scale up, so they can commercialize the products seamlessly. Rapid access may also pave the way for quicker evaluation of reagents from other laboratories that could become part of the drug development process, ranging from target identification through to medical chemistry areas to clinical. And by networking with the 


\section{Box 1 No startup alternative?}

It's unlikely that reagent development would be the basis of a venture capital-backed startup company. There are a hundred companies, for example, many in China, that make reagents and small building blocks. "It's more of a meat-and-potatoes business," says Scripps' Paul Schimmel. "It does not have the same sex appeal as the discovery of a new protein biologic or a new signaling pathway that could be applied to disease."

Scripps did contemplate the notion of a reagent startup, according to Scott Forrest. But after a fair amount of diligence, it saw that inventory would be an issue-most reagent companies need to be able to fill orders quickly, and therefore need a substantial inventory. "At the time, we did not wish to invest ourselves in building such an inventory 'on spec'," he says.

That said, reagent tools may be closely aligned to the development of new chemical entities for drug development, and there are examples of venture capitalists backing companies that have developed a new kind of chemistry.

For example, Ben Cravatt, whose laboratory is covered under the Sigma-Aldrich deal, years ago developed a way to label active sites of broad classes of proteins. Such reagents could be useful to test how to kill the activity of an enzyme or modify it so that it is not toxic. Cravatt formed a company in La Jolla (ActivX Biosciences) around that work. "That business [is] very good for identifying off-target activities of drugs that are ready to go into the clinic," says Schimmel, who was also a co-founder.

researchers at Scripps, Halford hopes to gather information for future products and services.

Several reagent categories were ready to go upon execution of the agreement. Only the discoveries from the Baran and Sharpless laboratories have been disclosed so far. "The rest will become public information in short order," says Forrest. "Look for publications out of those groups and you'll find the catalog numbers that correspond to the reagents we are rolling out."

In addition to chemical aids to drug development, compounds already superseded by proprietary lead drug development candidates are contemplated under the agreement. "We should be disclosing some of those as well, soon," Forrest says. "We will publish with a tool-like compound but will have in addition to that a family of IP [intellectual property] covering potential therapeutic candidates that are separate and distinct and not the subject of this agreement."

Reagents have largely been "a wasting asset" in academia, says Scripps' Paul Schimmel, whose laboratory is not focused on chemical reagent development and is not included in the deal. But universities are realizing more and more that these chemical tools are assets that can be utilized for research which is beneficial not just to the university and its partner, but "helps fulfill the mission of transferring knowledge to society in a way that enhances productivity and economic growth," he says. That is particularly important with the downturn in academic research funding, says Schimmel.

The dicey funding climate both within the National Institutes of Health (NIH) and for the internal research units of big pharma "has driven academia and pharma into each other's arms in ways they never really imagined ten years ago," says Forrest. Deal structures such as New York-based Pfizer's with the University of California, San Francisco, and later several other research institutions, are a model not unlike the Scripps-Sigma-Aldrich arrangement (Nat. Biotechnol. 29, 3, 2011).

But lack of experience is leading academic and not-for-profit institutions to accept weaker and weaker deal terms with pharma. "That is one of the negatives of the macroenvironment," says Forrest. Scripps, however, has proved to be among the most experienced and agile life sciences not-for-profits in dealing with industry.

For 30 years, Scripps held to a so-called firstrights partnership paradigm, where one company at a time would come in, pay somewhere on the order of \$20-25 million a year, and as a result have some first rights to technology, to sponsor research programs, and to provide materials under materials transfer agreements for Scripps researchers doing studies with drugs and other industry tools. In many cases, research support goes to specific investigators, making it difficult for institutions to fund general infrastructure such as support services and equipment used throughout the institution. But Scripps was able to accomplish this by licensing out broad rights. It had several serial mega-agreements with big pharma, including deals with Holzkirchen, Germanybased Sandoz, Basel-based Novartis and most recently, Pfizer.

The Scripps deal with Pfizer ended in 2011. At that same time, Scripps transitioned presidential leadership from Richard Lerner, who ran the institute very successfully for 25 years, to Michael Marletta. "Between new leadership's philosophy of how we engage industry and the 


\section{IN brief}

\section{Elan's Irish tax allure}

Dublin-based biotech Elan in July agreed to a takeover bid from US drug maker Perrigo in a deal worth $\$ 8.6$ billion. The buyout gives Perrigo a royalty stream from Tysabri (natalizumab), a blockbuster multiple sclerosis drug developed by Elan. The deal also allows Perrigo to move its domicile from Allegan, Michigan, to Ireland, where there is a comparatively lower corporate tax rate. That bonus likely drove the acquisition, says Eric Schmidt, an analyst with Cowen Group in New York. "Elan is not worth the price that Perrigo is paying," Schmidt says. "So I have to believe that Perrigo is willing to pay the higher price because of the tax benefit." Perrigo, the largest maker of generic drugs for major US retailers, will likely see its tax rate drop from $30 \%$ to the high teens, according to the company. Elan positioned itself for an acquisition earlier this year when it sold much of its stake in Tysabri to its partner, Biogen Idec in Weston, Massachusetts. Biogen paid Elan $\$ 3.25$ billion for the full rights to the drug and agreed to pay royalties on sales. "Elan said it would reinvest the proceeds [from the Tysabri sale] and investors were uncomfortable with that," says Cowen. "That put a lot of pressure on them to be sold." Elan's board in May rejected a bid from Royalty Pharma for $\$ 13$ cash per share plus $\$ 2.50$ more contingent upon Tysabri sales milestones. Perrigo's bid gives Elan's shareholders $\$ 6.25$ in cash and 0.076 of a Perrigo share for each Elan share. Elan shareholders are expected to vote on the acquisition by the end of the year. Emily Waltz

\section{IN their words}

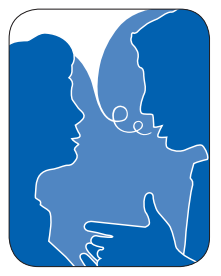

"Why call off the World Series when there's no rain and everybody's healthy, and the stadium is full of people?" George Church, commenting on the decision by organizers to cancel the \$10-million Archon Genomics X-Prize, a competition to sequence 100 genomes, in 30 days, at less than $\$ 10,000$ per genome, just two weeks before it was due to start. (LA Times, 24 August 2013)

"The royalty play illustrates the 'new normal' in life sciences VC investing." Steve Dickman opines on the move by some VC firms into investing in royalties in search of faster returns and sure-fire deals. (Xconomy, 16 August 2013)

"You get these windows, you get companies funded, you get shareholders that are interested in an approach, a strategy, a technology, a management team, and often, you really get big winners." Sam Waksal who, after serving jail time, and although banned for life from operating a publicly traded company for insider trading in 2003, is looking to China for his latest spin-out, the gene therapy firm Kadmon. (Bloomberg, 3 September 2013) changing, shifting sands on the industry side, [we decided] our interaction could have been more focused," says Forrest.

"We are trying to reach out and bridge the basic research that is starting to produce a lot of commercially useful assets, while retaining academic independence," says Schimmel. In addition to Sigma-Aldrich, Scripps now has focused collaborations with Crucell, in Leiden, The Netherlands, for vaccine development, and with New York-based Bristol-Myers Squibb around synthesis of new drug candidates. Under this scheme, there's involvement and ownership of the work plan by both senior leadership at Scripps and at the companies, Forrest says. The Sigma-Aldrich deal fits the category. With no lingering first-rights deals, Scripps is also looking more to spin out more disruptive projects as startup companies, although it has no such plans in the reagent development space (Box 1).

Among the academic community, Baran notices much more willingness to collaborate with industry. "When you work with a company there's more accountability. If you do a good job you can be assured your funding will continue. With grant money from NIH, however, no such safety net exists," Baran says. He urges companies to "strike while the iron is hot," adding that, "the academic community will be more receptive than when the NIH pay line is high." It's rewarding on multiple levels: students are inspired to work more diligently when they can say they are working on something funded by a company instead of taxpayer money, he says.

Indeed, students are emotionally affected by "the overhang of negativity that is out there now in terms of the lack of support for research and the lack of society interest in research and graduate education," adds Schimmel. "That drives a lot of students out of the field." But agreements such as the one with Sigma-Aldrich, that show the utility of what they are doing, are "a real morale booster," he says.

Mark Ratner Cambridge, Massachussetts

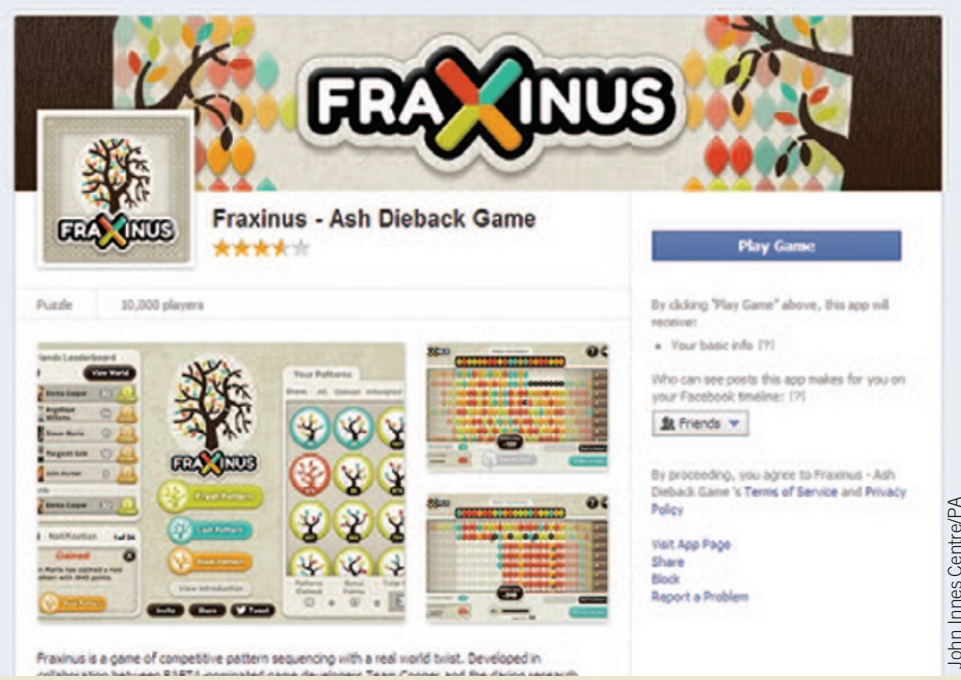

Facebook gamers race against fungal menace.

Thousands of citizen scientists logged on to Fraxinus in August to help save the UK's ancient ash woodlands. The game aims to discover genetic variants that resist the fungus Chalara fraxinea, known as ash dieback. Scientists at the Sainsbury Laboratory, University of Cambridge, have identified 100 trees from Denmark that have resisted the disease. The gamers will be matching on-screen patterns that correlate with genetic data. They will be comparing patterns from trees that resist with those that succumb to identify DNA sequences most likely to be useful in breeding programs. 